\title{
The clinical ability of contrast-enhanced magnetic resonance imaging to predict treatment outcomes for lumbar facet joint pain
}

\author{
Min Cheol Chang ${ }^{1} \wedge$, You Gyoung Yi ${ }^{2}$, Hea-Eun Yang ${ }^{2}$, Jang Ho Lee ${ }^{2}$, Ji Hwan Kim², Kyung Hee Do ${ }^{2 \wedge}$ \\ ${ }^{1}$ Department of Physical Medicine and Rehabilitation, College of Medicine, Yeungnam University, Daegu, Korea; ${ }^{2}$ Department of Physical Medicine \\ and Rehabilitation, Veterans Health Service Medical Center, Seoul, Korea \\ Contributions: (I) Conception and design: KH Do, MC Chang; (II) Administrative support: MC Chang; (III) Provision of study materials or patients: \\ MC Chang; (IV) Collection and assembly of data: MC Chang; (V) Data analysis and interpretation: KH Do, YG Yi, HE Yang; (VI) Manuscript \\ writing: All authors; (VII) Final approval of manuscript: All authors. \\ Correspondence to: Kyung Hee Do, MD, PhD. Department of Physical Medicine and Rehabilitation, Veterans Health Service Medical Center, Seoul, \\ Korea. Email: khdo79@gmail.com.
}

\begin{abstract}
Background Several radiologic imaging techniques have been used to predict the effects of treatment on lumbar facet joint (LFJ) pain. However, there are no reports on the use of contrast-enhanced magnetic resonance imaging (MRI) in the management of LFJ pain. In the current study, we aimed to evaluate the clinical ability of contrast-enhanced MRI to predict treatment outcomes for LFJ pain.

Methods: A total of 26 patients with LFJ pain were recruited and intraarticular (IA) corticosteroid injections were administered to each patient. We assessed the treatment outcomes using a numerical rating scale (NRS), and two radiologists independently investigated LFJ enhancement and osteoarthritis grading. No serious complications or adverse events were reported.
\end{abstract}

Results: IA corticosteroid injections were administered to 26 patients (12 women and 14 men; mean age: $65.19 \pm 11.05$ years) with LFJ pain. Among the 26 patients, 16 patients were included in the facet joints with enhancement group, and the remaining 10 patients were included in the facet joints with non-enhancement group, based on contrast-enhanced MRI scans. In both the enhancement and non-enhancement groups, NRS scores significantly decreased at 1,2 , and 3 months after treatment $(\mathrm{P}<0.05)$. However, we saw no significant difference between the groups from pretreatment to three months after treatment $(\mathrm{P}>0.05)$.

Conclusions: The routine use of contrast-enhanced MRI is not recommended in patients with LFJ pain.

Keywords: Magnetic resonance imaging (MRI); image enhancement; zygapophyseal joint; low back pain; injection; intraarticular injection (IA injection); treatment

Submitted Feb 04, 2020. Accepted for publication Sep 18, 2020.

doi: 10.21037/apm-20-313

View this article at: http://dx.doi.org/10.21037/apm-20-313

\section{Introduction}

Radiologic imaging techniques can identify a given source of pain or distinguish it from other possible sources of pain, however, it is controversial especially in patients with lumbar facet joint (LFJ) disease (1-4). This discrepancy results from degenerative changes found in patients with
LFJ disease that are frequently also found in asymptomatic patients, making it difficult to clearly identify sources of pain (1-4). Therefore, proper assessment of LFJs is important for patients with LFJ pain.

The effectiveness of several radiologic imaging techniques, including radiographs, computed tomography

^ ORCID: Min Cheol Chang, 0000-0002-7629-7213; You Gyoung Yi, 0000-0003-1791-1831; Hea-Eun Yang, 0000-0002-4449-7288; Kyung Hee Do, 0000-0003-4235-8759. 
(CT), single-photon emission CT (SPECT), and magnetic resonance imaging (MRI), have been reported by comparing the presence of osteoarthritis changes to the treatment outcomes for patients with LFJ pain $(1,5-8)$. However, there has not yet been any study to report on the clinical ability of contrast-enhanced MRI to predict the effects of treatment for LFJ pain.

In this study, we aimed to evaluate the correlation between contrast-enhanced MRIs and treatment outcomes for LFJ intraarticular (IA) steroid injections. We present the following article in accordance with the MDAR reporting checklist (available at http://dx.doi.org/10.21037/apm-20313).

\section{Methods}

\section{Study design}

The patients were recruited retrospectively and consecutively based on injection charts of the spine center of a university hospital between March 2012 and September 2018 according to the following criteria: lumbar axial pain persisting for more than six months without radicular symptoms; age: $21-79$ years; more than three on the numerical rating scale (NRS); a minimum of $80 \%$ temporary pain improvement for a minimum of 30 minutes after a selective diagnostic block with $0.5 \mathrm{~mL}$ of $1 \%$ lidocaine to each LFJ pain location (9); patients who had been conducted contrast-enhanced lumbar spine MRI that showed spondyloarthropathy; lower lumbar spine pain that increased during rotation, lateral bending, and hyperextension; and local paraspinal tenderness. The exclusion criteria for the current study were patients with allergy to contrast materials, spinal instability, coagulopathy, any uncontrolled psychiatric or medical condition, and rheumatic diseases. Also, in case of the patients with disc herniation or lumbar spinal stenosis, the patients whose pain did not match with the characteristics of the LFJ pain were excluded. The study was conducted in accordance with the Declaration of Helsinki (as revised in 2013). The institutional review board of Yeungnam University Hospital approved the current study protocol (Clinical Trial Registry Number: 2017-02-011) and individual consent for this retrospective analysis was waived.

Patients were classified into facet joints with enhancement and non-enhancement groups, based on contrast-enhanced MRI scans with gadolinium (Figure 1). MRI data were obtained using a 1.5-T scanner (Magnetom Vision, Siemens, Erlangen, Germany) with a spine array coil. Spin-echo sequences, axial and sagittal T1- [583/12 (repetition time ms/echo time ms)], turbo T2-weighted images (3800/128), and contrast (Magnevist), $0.2 \mathrm{~mL} / \mathrm{kg}$ of gadopentetate dimeglumine (Bayer Healthcare Pharmaceuticals) enhanced axial T1-weighted images were obtained. The grading criteria for osteoarthritis of the facet joint was also used, and four categories are shown as follows: zero indicated normal facet joint space ( $2-4 \mathrm{~mm}$ width); one indicated narrowing of the facet joint space $(<2 \mathrm{~mm})$, and/ or presence of small osteophytes, and/or mild hypertrophy of the articular process; two indicated narrowing of the facet joint space, and/or presence of moderate osteophytes, and/or moderate hypertrophy of the articular process, and/
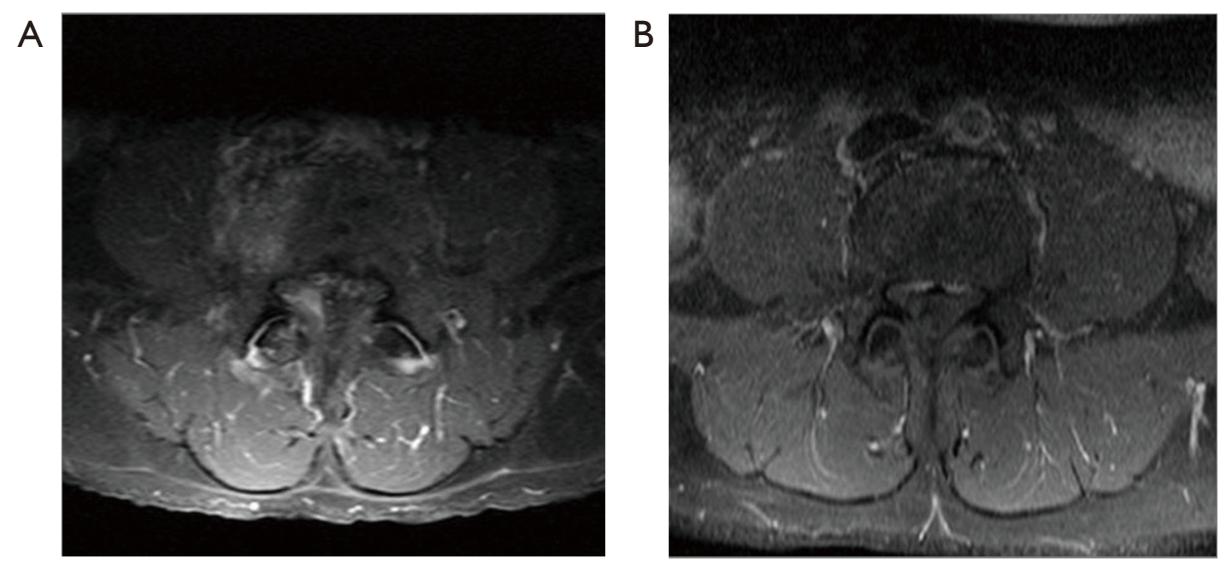

Figure 1 Enhancement and non-enhancement of the lumbar facet joint in axial contrast-enhanced T1 magnetic resonance imaging. (A) Enhanced lumbar facet joint; (B) non-enhanced lumbar facet joint. 
Table 1 Demographic and clinical data of the patients

\begin{tabular}{|c|c|c|c|}
\hline Variable & $\begin{array}{l}\text { Facet joins with enhancement } \\
\text { group }\end{array}$ & $\begin{array}{l}\text { Facet joints with non-enhancement } \\
\text { group }\end{array}$ & $P$ value \\
\hline Sex (male/female) & $9 / 7$ & $5 / 5$ & 1 \\
\hline Duration until treatment from pain onset (months) & $29.25 \pm 28.19$ & $33.30 \pm 46.22$ & 0.689 \\
\hline
\end{tabular}

Values are shown as number or mean \pm standard deviation. *, $\mathrm{P}<0.05$. Facet degeneration grades are graded by criteria for grading osteoarthritis of the lumbar facet joints on T2-weighted imaging (10).

or mild subarticular bone erosions; and three indicated narrowing of the facet joint space, and/or presence of large osteophytes, and/or severe hypertrophy of the articular process, and/or severe subarticular bone erosions, and/or subchondral cysts (10). Two radiologists, who were unaware of the treatment results, independently assessed the LFJ enhancement and osteoarthritis grading.

An aseptic IA corticosteroid injection was administered using C-arm fluoroscopic guidance (Siemens, Erlangen, Germany) with a posterior approach. The IA injection was performed at the same one level as the selective diagnostic block. After the patient was lying down in a prone position using a comfortable pillow under the abdomen to straighten the lower lumbar spine, the C-arm fluoroscopy was placed in an oblique and cephalad position until the LFJ space could be seen clearly. After targeting the end point of the superior articular process as an injection point, a $90-\mathrm{mm}$, 26-gauge spinal needle was positioned to the superior articular process parallel to the beam of the $\mathrm{C}$-arm fluoroscopy. Then, an IA injection of $0.3 \mathrm{~mL}$ of contrast material was administered to confirm correct IA LFJ access. After that, $10 \mathrm{mg}(0.25 \mathrm{~mL})$ of dexamethasone with $0.5 \mathrm{~mL}$ of $0.25 \%$ bupivacaine was injected to the LFJs. A single skillful physician with more than 10 years of experience performed these injections.

We measured clinical outcomes using an NRS score to evaluate pain intensity (11). Outcome assessments were performed prior to treatment and at 1,2, and 3 months after treatment. Third parties who were unaware of the demographic and clinic data of the patients conducted these assessments.

\section{Statistical analysis}

To analyze the data, we performed statistical analyses using PASW Statistics for Windows, Version 18.0 (SPSS Inc.,
Chicago, IL, USA). Independent sample $t$-tests were used to compare the patients' demographic and clinical data, excluding the non-parametric data. A Fisher's exact test was used to analyze the non-parametric data, including sex and facet degeneration grade. We performed a one-way analysis of variance for repeated measurements, followed by a post hoc analysis with a Bonferroni correction to analyze changes in NRS score for each group. In addition, we performed comparative analyses to identify differences in NRS scores between the two groups. We used a $\mathrm{P}$ value threshold of $<0.05$ to define statistical significance.

\section{Results}

A total of 26 patients (12 women and 14 men; mean age: $65.19 \pm 11.05$ years) were retrospectively recruited for this study. Demographic and clinical data for the patients are presented in Table 1. Among the 26 patients, 16 patients were included in the facet joints with enhancement group, and the remaining 10 patients were included in the facet joints with non-enhancement group. Out of all variables, only the mean age of patients showed a significant difference between the two groups $(\mathrm{P}<0.05)$. Further, we did not observe any significant complication or adverse event after the procedures.

In the group comparison, both groups showed significantly improved NRS scores after treatment $(\mathrm{P}<0.05$, Figure 2, Table 2). In detail, the enhancement group showed decreased NRS scores from $4.36 \pm 0.81$ before treatment to $2.50 \pm 1.26,2.38 \pm 1.36$, and $2.38 \pm 1.41$ at 1,2 , and 3 months after treatment, respectively $(\mathrm{P}<0.05)$. The mean NRS scores of the non-enhancement group also decreased from $4.60 \pm 1.26$ before treatment to $2.10 \pm 1.52,2.00 \pm 1.76$, and $2.30 \pm 1.83$ at 1,2 , and 3 months after treatment, respectively $(\mathrm{P}<0.05)$. We also compared the treatment outcomes between two groups; however, we saw no significant 


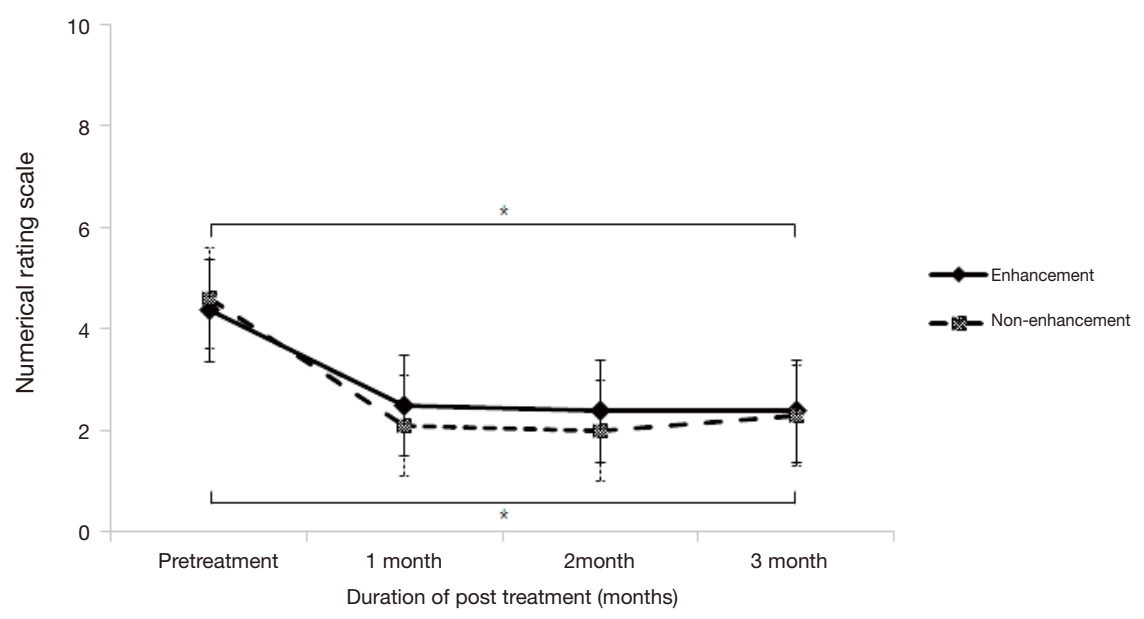

Figure 2 Comparative results of numerical rating scale of lumbar facet joint pain in facet joints with enhancement and non-enhancement groups. *, $\mathrm{P}<0.05$.

Table 2 Changes in numerical rating scale scores from pretreatment to 3 months after treatment

\begin{tabular}{lccc}
\hline NRS & $\begin{array}{c}\text { Facet joints with } \\
\text { enhancement group }\end{array}$ & $\begin{array}{c}\text { Facet joints with non- } \\
\text { enhancement group }\end{array}$ & P value (group comparison) \\
\hline Pretreatment & $4.36 \pm 0.81$ & $4.60 \pm 1.26$ & 0.746 \\
After 1 month of treatment & $2.50 \pm 1.26$ & $2.10 \pm 1.52$ \\
After 2 months of treatment & $2.38 \pm 1.36$ & $2.00 \pm 1.76$ \\
After 3 months of treatment & $2.38 \pm 1.41$ & $2.30 \pm 1.83$ \\
Time effect & $<0.001^{*}$ & $<0.001^{*}$
\end{tabular}

Values are shown as number or mean \pm standard deviation. ${ }^{*}, \mathrm{P}<0.05 . \mathrm{NRS}$, numerical rating scale, time effect, temporal changes of NRS scores in each group after treatment.

difference between the enhancement and non-enhancement groups from pretreatment to 3 months after treatment $(\mathrm{P}=0.746)$.

There was $96.1 \%$ agreement between the two radiologists about the enhancement and $92.3 \%$ agreement about grading for osteoarthritis of the LFJs.

\section{Discussion}

In this study, we evaluated the clinical ability of contrastenhanced MRI scans to predict the effects of treatments for LFJ pain. The results of this study showed that the use of contrast-enhanced MRIs did not correlate with a positive treatment response to IA corticosteroid injection in patients with LFJ pain.

Many radiologic imaging techniques have been used to diagnose LFJ pain, and MRI is a nonionizing and noninvasive method that produces images with a good soft tissue resolution $(12,13)$. MRIs have an excellent advantage for evaluating the immediate consequences of LFJ degeneration, including adjacent bone edema, active synovial inflammation and neural structure impingement, enhancement of the LFJ rim, LFJ effusion, subchondral bone edema, and wraparound bumper osteophyte formation $(12,13)$. In addition, some studies have reported that the enhancement of the LFJ rim with gadolinium can lead to more accurate diagnoses for synovitis $(12,13)$.

Some studies asserted that these various radiologic imaging studies can predict treatment responses $(6,8)$, and others cannot $(1,5,7)$. In 1984, Carrera and Williams administered IA LFJ injections using $15 \mathrm{mg}$ depo-methylprednisolone acetate and $2-4 \mathrm{~mL}$ of $1 \%$ lidocaine solution 
to 63 patients, and they found that $73 \%$ of the observed pain relief occurred when the CT scan of the patient showed LFJ disease (6). However, only $13 \%$ of the observed pain relief occurred when the CT scan of the patient showed no definite pathology, so they reported that CT scans are likely effective for predicting treatment effect (6). Pneumaticos et al. [2006] evaluated the results of LFJ injections according to positive response to bone scintigraphy in 47 patients with low back pain (8). The patients with the positive SPECT findings presented better treatment outcomes, so they asserted that bone scintigraphy with SPECT can be used to give information about who would most benefit from LFJ injections (8). However, Jackson et al. [1988] reported no correlation between lumbar radiographs of LFJ and response to single IA LFJ injection with $20 \mathrm{mg}$ of triamcinolone and $1 \mathrm{~mL}$ of $0.5 \%$ bupivacaine hydrochloride in 390 patients, so they reported that lumbar radiographs are likely ineffective for predicting treatment effect (5). Schwarzer et al. [1995] also conducted a placebo-controlled study, which showed no correlations between CT findings and a positive response to local anesthetics in 63 patients (1). Cohen $e t a l$. [2007] evaluated the clinical ability to predict the treatment outcome of a medial branch radiofrequency according to a positive response to a single medial branch block in 192 patients (7). They found no correlation between the non-enhanced MRI findings, such as facet hypertrophy or degeneration, and treatment outcome after six months (7).

To our knowledge, previous studies on the correlation between contrast-enhanced MRIs and treatment outcomes for LFJ injections have been limited and this is the first study which investigates the clinical ability of contrastenhanced MRI to predict the effects of treatment for LFJ pain. Further encouragement for our study was supported by a previous study by D'Aprile et al. [2006], where they evaluated the treatment potential of MR sequences using T2-weighted sequences with fat saturation and gadoliniumadministered T1-weighted sequences with fat saturation in 3,323 patients with low back pain and degenerative lumbar disease (14). They asserted that these imaging examinations were advantageous for showing inflammatory and degenerative changes in the lumbar spine because these can evaluate the active inflammatory processes, which may not be disclosed by using a standard MRI (14). Therefore, we tried to investigate the clinical ability of contrast-enhanced MRI to predict the effects of treatment effect for LFJ pain in the current study. However, the results of our study showed that contrast-enhanced MRI did not correlate with a positive treatment outcome in patients with LFJ pain. However, we can possibly suggest some hypotheses about some positive treatment effects of a selective diagnostic block to each LFJ pain location, and these could make no correlation between contrast-enhanced MRI findings and LFJ steroid injection outcomes.

Considering the demographic and clinical data for the patients of the current study, the enhancement group had a significantly older age than the non-enhancement group. These results are consistent with previous studies, which showed that the prevalence of abnormal changes of the LFJ on radiologic imaging increased with age due to its similarity to arthritis (15-20). In 1964, Lewin evaluated that the LFJ showed only minor chondral changes for patients aged younger than 45 years, and the incidence of osteophytes, subchondral sclerosis, and advanced chondral changes increased with older age (17). Fujiwara et al. [1999] studied $84 \mathrm{LFJ}$ cases for 14 patients with degenerative disc disease, and they revealed that the prevalence of degenerative changes for LFJ was minimal in patients before the age of 40 . However, these changes significantly increased in patients aged older than 60 years, but these changes were not always general (20). Kalichman et al. [2010] revealed that $25.0 \%$ of LFJ osteoarthritis cases, including hypertrophy of the articular process, osteophytes, subchondral cysts, subarticular sclerosis, joint space narrowing, and the vacuum phenomenon, were observed in CT scans for patients that were aged younger than 40 years and $87.5 \%$ were observed in patients aged over 60 years (19). In 2012, DePalma et al. conducted a retrospective chart review using multivariable analyses in 157 cases from 153 patients and they evaluated that degenerative spinal changes were more frequently seen in patients of older ages (16). In detail, for male patients who were aged approximately 65 years, degenerative spinal changes associated with LFJ were more frequently related to increased age regardless of BMI, while for female patients who were aged approximately 65 years, these change occurred usually when BMI was 30 or $35 \mathrm{~kg} / \mathrm{m}^{2}$ (16). Therefore, in the current study, we saw an agreement with this hypothesis in that the enhancement group showed a significantly higher older age than the nonenhancement group.

This study is the first trial evaluating the correlation between contrast-enhanced MRIs and treatment outcomes for LFJ IA steroid injections. Our study found no correlation between contrast-enhanced MRI findings and LFJ steroid injection outcomes. Therefore, the authors suggested that the routine use of contrast-enhanced MRI is 
not recommended in patients with LFJ pain. However, the current study was performed retrospectively with a small number of patients without calculation of sample size, so larger studies with randomized control are recommended for the future. In addition, further clinical outcome measures, including patient functionality or quality of life are recommended.

\section{Acknowledgments}

Funding: This study was supported by a VHS Medical Center Research Grant, Republic of Korea (grant number: VHSMC18019). Also, this study was supported by a National Research Foundation of Korea grant funded by the Korean government (grant number: NRF2019R1F1A1061348).

\section{Footnote}

Reporting Checklist: The authors have completed the MDAR reporting checklist. Available at http://dx.doi.org/10.21037/ apm-20-313

Data Sharing Statement: Available at http://dx.doi. org/10.21037/apm-20-313

Peer Review File: Available at http://dx.doi.org/10.21037/ apm-20-313

Conflicts of Interest: All authors have completed the ICMJE uniform disclosure form (available at http://dx.doi. org/10.21037/apm-20-313). The authors have no conflicts of interest to declare.

Ethical Statement: The authors are accountable for all aspects of the work in ensuring that questions related to the accuracy or integrity of any part of the work are appropriately investigated and resolved. The study was conducted in accordance with the Declaration of Helsinki (as revised in 2013). The institutional review board of Yeungnam University Hospital approved the current study protocol (Clinical Trial Registry Number: 2017-02-011) and individual consent for this retrospective analysis was waived.

Open Access Statement: This is an Open Access article distributed in accordance with the Creative Commons Attribution-NonCommercial-NoDerivs 4.0 International
License (CC BY-NC-ND 4.0), which permits the noncommercial replication and distribution of the article with the strict proviso that no changes or edits are made and the original work is properly cited (including links to both the formal publication through the relevant DOI and the license). See: https://creativecommons.org/licenses/by-nc-nd/4.0/.

\section{References}

1. Schwarzer AC, Wang SC, O'Driscoll D, et al. The ability of computed tomography to identify a painful zygapophysial joint in patients with chronic low back pain. Spine (Phila Pa 1976) 1995;20:907-12.

2. Lawrence JS, Bremner JM, Bier F. Osteo-arthrosis. Prevalence in the population and relationship between symptoms and $x$-ray changes. Ann Rheum Dis 1966;25:1-24.

3. Magora A, Schwartz A. Relation between the low back pain syndrome and $\mathrm{x}$-ray findings. I. Degenerative osteoarthritis. Scand J Rehabil Med 1976:115-25.

4. Wiesel SW, Tsourmas N, Feffer HL, et al. A study of computer-assisted tomography. I. The incidence of positive CAT scans in an asymptomatic group of patients. Spine (Phila Pa 1976) 1984;9:549-51.

5. Jackson RP, Jacobs RR, Montesano PX. 1988 Volvo award in clinical sciences. Facet joint injection in low-back pain. A prospective statistical study. Spine (Phila Pa 1976) 1988;13:966-71.

6. Carrera GF, Williams AL. Current concepts in evaluation of the lumbar facet joints. Crit Rev Diagn Imaging 1984;21:85-104.

7. Cohen SP, Hurley RW, Christo PJ, et al. Clinical predictors of success and failure for lumbar facet radiofrequency denervation. Clin J Pain 2007;23:45-52.

8. Pneumaticos SG, Chatziioannou SN, Hipp JA, et al. Low back pain: prediction of short-term outcome of facet joint injection with bone scintigraphy. Radiology 2006;238:693-8.

9. Manchikanti L, Pampati S, Cash KA. Making sense of the accuracy of diagnostic lumbar facet joint nerve blocks: an assessment of the implications of $50 \%$ relief, $80 \%$ relief, single block, or controlled diagnostic blocks. Pain Physician 2010;13:133-43.

10. Weishaupt $\mathrm{D}$, Zanetti $\mathrm{M}$, Boos $\mathrm{N}$, et al. MR imaging and $\mathrm{CT}$ in osteoarthritis of the lumbar facet joints. Skeletal Radiol 1999;28:215-9.

11. Hartrick CT, Kovan JP, Shapiro S. The numeric rating scale for clinical pain measurement: a ratio measure? Pain 
Pract 2003;3:310-6.

12. Perolat R, Kastler A, Nicot B, et al. Facet joint syndrome: from diagnosis to interventional management. Insights Imaging 2018;9:773-89.

13. Clarencon F, Law-Ye B, Bienvenot P, et al. The Degenerative Spine. Magn Reson Imaging Clin N Am 2016;24:495-513.

14. D'Aprile P, Tarantino A, Lorusso V, et al. Fat saturation technique and gadolinium in MRI of lumbar spinal degenerative disease. Neuroradiol J 2006;19:654-71.

15. Cohen SP, Raja SN. Pathogenesis, diagnosis, and treatment of lumbar zygapophysial (facet) joint pain. Anesthesiology 2007;106:591-614.

16. DePalma MJ, Ketchum JM, Saullo TR. Multivariable analyses of the relationships between age, gender, and body mass index and the source of chronic low back pain.
Pain Med 2012;13:498-506.

17. Lewin T. Osteoarthritis in Lumbar Synovial Joints. A Morphologic Study. Acta Orthop Scand Suppl 1964:SUPPL 73:1-112.

18. Pollintine P, Dolan P, Tobias JH, et al. Intervertebral disc degeneration can lead to "stress-shielding" of the anterior vertebral body: a cause of osteoporotic vertebral fracture? Spine (Phila Pa 1976) 2004;29:774-82.

19. Kalichman L, Kim DH, Li L, et al. Computed tomography-evaluated features of spinal degeneration: prevalence, intercorrelation, and association with selfreported low back pain. Spine J 2010;10:200-8.

20. Fujiwara A, Tamai K, Yamato $M$, et al. The relationship between facet joint osteoarthritis and disc degeneration of the lumbar spine: an MRI study. Eur Spine J 1999;8:396-401.

Cite this article as: Chang MC, Yi YG, Yang HE, Lee JH, Kim JH, Do KH. The clinical ability of contrast-enhanced magnetic resonance imaging to predict treatment outcomes for lumbar facet joint pain. Ann Palliat Med 2021;10(2):1431-1437. doi: 10.21037/apm-20-313 